\title{
Acetylcholinesterase activity and menstrual remissions in myasthenia gravis
}

\author{
N. V I J A Y A N, V.K. VI J A Y A N, A N D P. M. D R E Y F U S \\ From the Departments of Neurology and Human Anatomy, School of Medicine, \\ University of California, Davis, California, USA
}

S UMMARY Menstrually related temporary remissions of myasthenic symptoms are reported to occur in 25 to $50 \%$ of female patients. Even though this has been attributed to hormonal changes associated with the menstrual cycle the underlying mechanism of this hormonal influence has remained elusive. The present study demonstrated a cyclical variation in the activity of red cell acetylcholinesterase (EC 3.1.1.7) enzyme (AChE) with a marked reduction at the time of menstrual remission of symptoms of myasthenia. These cyclical changes were abolished by thymectomy. It appears, therefore, that menstrual remission in myasthenia is at least partly due to hormone-induced changes in AChE activity. This process seems to be under the control of the thymus gland.

Temporary exacerbation and remission of symptoms are fairly frequent in patients with myasthenia gravis. Exacerbations can often be attributed to readily identifiable causes such as excessive physical activity, intercurrent infection, emotional stress, pregnancy, or change in medications. At other times no specific precipitating factors are obvious. Similarly, temporary remissions may occur spontaneously, during pregnancy, or at the beginning of each menstrual cycle (Viets et al., 1942; Keynes, 1952). Naturally the exacerbations and remissions seen in pregnancy and menstruation have been attributed to associated endocrine changes. This has prompted investigators to study the relationship between myasthenia gravis and the endocrine system. However, the mechanism underlying these changes has remained elusive.

Menstrual remission of symptoms was observed in three of 12 women in our myasthenia gravis clinic. Red cell acetylcholinesterase (EC 3.1.1.7; AChE) activity was studied in two of these patients during five menstrual cycles.

Address for reprint requests: Dr N. Vijayan, Department of Neurology, School of Medicine, University of California, Davis, California 95616, USA.

Accepted 19 July 1977

\section{Patients and methods}

CASE 1

This 30 year old woman developed weakness of limbs at age 25 years. This symptom gradually became generalised, involving speech, chewing, swallowing, and occasionally breathing. There were no ocular symptoms. Examination at age 30 years revealed generalised muscular weakness with more severe involvement of proximal muscles. There was bilateral facial weakness and ptosis. Edrophonium test and repetitive nerve stimulation studies confirmed the diagnosis of myasthenia gravis. She was treated with pyridostigmine, $90 \mathrm{mg}$ every three to four hours.

Menstrual history Menarche was normal and the menstrual cycle had been quite regular from the onset. A clear-cut relationship between the menstrual cycle and severity of myasthenic symptoms was observed by the patient from the very beginning of the disease. She reported mild improvement of symptoms 12 to 24 hours before menstrual flow, followed by a marked improvement with the onset of the flow. This improvement persisted for the next five to six days. Her medication requirement was lower during this time. Gradually her symptoms would get worse and she would have to take larger amounts of medication as the cycle progressed. Symptoms would be at their worst just before the onset of 
the next menstrual flow. This dramatic and close relationship persisted until thymectomy. After thymectomy, the cyclical variations, though still present, were not as marked as before.

\section{CASE 2}

A 15 year old girl first experienced a lumpy feeling in her throat and dysarthria. Within a few months she developed ptosis and diplopia. Soon, generalised muscle weakness became obvious. Examination revealed diffuse muscular weakness, bilateral ptosis, and limited ocular motility. An edrophonium test produced marked improvement. Repetitive nerve stimulation studies showed a decrementing muscular response diagnostic of myasthenia gravis. She was treated with anticholinesterase medications, with symptomatic improvement. (These cyclical changes have improved after a recent thymectomy.)

Menstrual history Menarche was at age 13 years and fairly regular menstrual periods occurred since then. The patient reported progressive worsening of myasthenic symptoms up to the beginning of the menstrual flow, when she noticed marked improvement of symptoms. The usual daily dosage of $360 \mathrm{mg}$ pyridostigmine caused toxic manifestations at the time of menstrual remission and had to be reduced to half that amount. This improvement usually persisted for four to five days and would then gradually wear off. Medication requirements would also go up gradually until the beginning of the next menstrual flow.

\section{CASE 3}

This patient was post-menopausal (46 years old) when first seen in the clinic. She had a long history of menstrual remissions of myasthenia since age 28 years when her symptoms first became evident. She had a mild generalised myasthenia gravis and had done very well on anticholinesterase medications. She used to require only a very small amount of medication (60 to $120 \mathrm{mg}$ of pyridostigmine daily) during the first half of her menstrual cycle. During the second half, however, she required two or three times that amount. With the onset of menstrual flow, she would become better. She was able to lead 'a fairly normal and active life for half of the month', in her own words. After the menopause these cyclical changes ceased and she required larger amounts of medication. She was unwilling to undergo thymectomy.

RED CELL AChE STUDIES

Red cell AChE was studied in patients 1 and 2 during five separate menstrual cycles before thymectomy. Two age- and race-matched control subjects were studied at the same time. Two myasthenics without menstrual remissions were also studied. During each cycle a total of nine blood samples were collected, five samples at three to four day intervals premenstrually and four daily samples during menstruation. Time of collection was approximately the same on every occasion. Patients were kept on a regular dosage schedule for the duration of the study, to avoid any changes in the enzyme activity due to the drug itself. Myasthenics were asked to stay off anticholinesterase medication for eight hours before collection of samples, however. No samples were collected if the patient could not stay off medication for a minimum of eight hours. This problem did not arise during menstruation as they invariably had milder symptoms. Control subjects were two young women who did not suffer from any menstrual or medical problems. Patients and controls were not on birth control pills.

Blood samples were collected in heparinised tubes with adequate precautions to avoid haemolysis. Red cells and serum were separated in a clinical centrifuge immediately after collection of the blood. Cells were washed three times with normal saline to remove all serum. Assaying of the red cell samples for serum cholinesterase (EC 3.1.1.8) helped to confirm that there was no serum contamination after this washing procedure. The washed red cells were haemolysed with 1.0 volume of distilled water and 0.4 volume of toluene. Red cell membranes were extracted twice after vigorous shaking and centrifugation at $6000 \mathrm{~g}$ for 20 minutes in a Sorvall refrigerated centrifuge. The cell membrane preparation was then resuspended to the original volume in $5 \%$ Triton $\mathrm{X}-100$ in $0.01 \mathrm{M}$ phosphate buffer at $\mathrm{pH}$ 7.4. This preparation was used for assay either immediately or after storage at $-10^{\circ} \mathrm{C}$ (Coates and Simpson, 1972). All estimations were done within seven days.

The assay procedure for AChE was that of Ellman et al. (1961), using acetylthiocholine iodide as substrate and dithiobisnitrobenzoic acid (DTNB) as the colouring agent. Specific inhibitors of AChE, ( $1: 5$-bis-[ 4 allyl dimethylammonium phenyl] pentan 3-1 dibromide; 284C51, Borroughs-Welcome) and cholinesterase (tetraisopropyl pyrophosphoramide; IsoOMPA, Sigma) were used. For the purpose of this study, red cell AChE values were expressed as change in absorbance/minute. Values were also calculated in terms of moles of substrate hydrolysed/min/ 
RBC. Assay of each sample was done in duplicate with and without the appropriate inhibitors.

\section{Results}

The data were analysed using the regressive analysis programmes BMDO1R and BMDO5R of the biomedical statistical package programmes. As expected from the small number of cases, most of the information was expressed by first degree regression. AChE levels during three menstrual cycles in the myasthenic patients and control subjects were fairly reproducible for each individual (Fig. 1). In the myasthenic patients there was a progressive increase in enzyme activity from the end of one menstrual cycle to the beginning of the next, when there was a rapid reduction in myasthenic patients the enzyme activity rose rapidly. Roughly halfway through the cycle, the enzyme levels increased above controls and continued to rise until the beginning of menstrual flow. The overall activity changed from 0.02 to $0.071 \Delta \mathrm{A} / \mathrm{min}$ in the myasthenics, compared to 0.052 to $0.066 \Delta \mathrm{A} / \mathrm{min}^{*}$ in controls. Myasthenic patients without menstrual remission of symptoms did not show this cyclical variation and resembled the normal controls.

These cyclical changes in AChE activity correlated well with the medication requirements (Fig. 3). This represents a single cycle in patient 1 , studied three months after thymectomy when the medication requirements had already diminished but the patient still experienced the cyclical variations, though to a lesser extent.

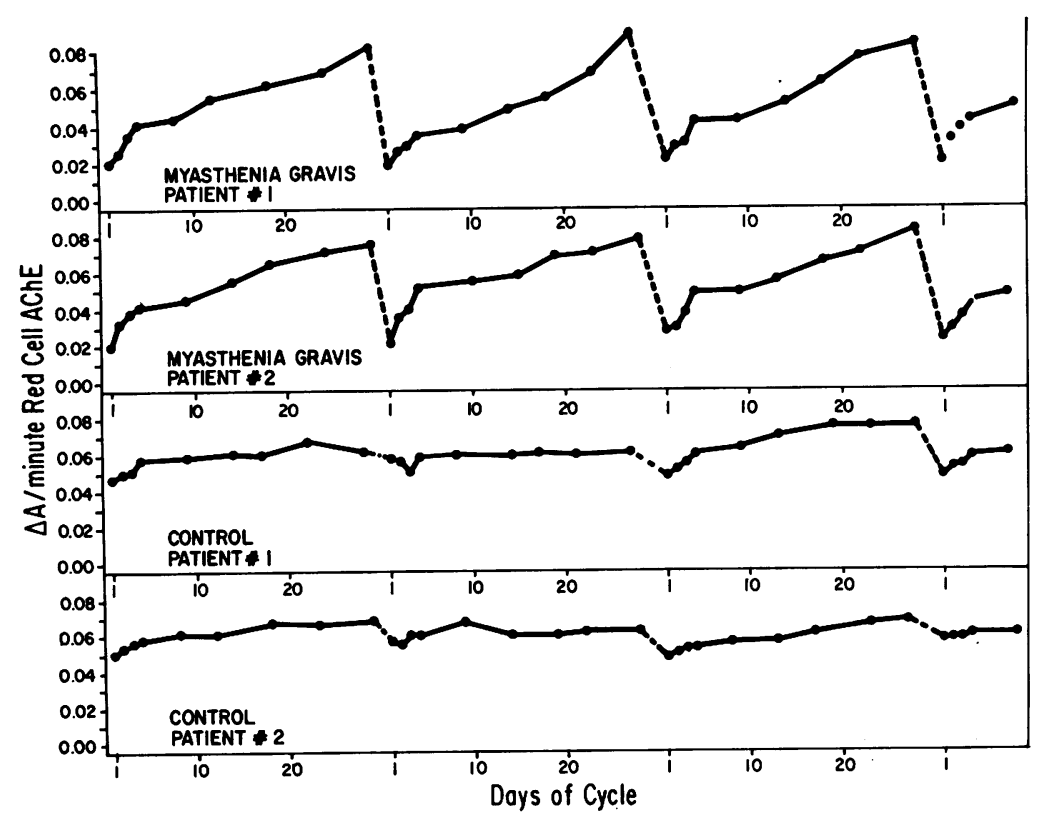

Fig. 1 Red cell AChE activity expressed as $\Delta A / \mathrm{min}$ during three menstrual cycles.

the activity. This cyclical change in enzyme activity was minimal in control subjects when compared with the patients.

In the quadratic regression curves (Fig. 2) the regression slopes of controls and myasthenic patients were significantly different ( $F$ ratio of 383.9 for 1.168 degrees of freedom). The control subjects showed almost straightline activity of the enzyme, with very little variation from the beginning to the end of the cycle. In myasthenic patients, the activity started at a lower level than controls, probably related to anticholinesterase medication. As the cycle progressed, however, in

\section{Discussion}

Menstrual influence on myasthenia gravis has been generally believed to be unpredictable or variable. Collins, in 1897, was the first to document menstrually related changes in myasthenic symptoms. Since then, detailed clinical observations of a similar nature have been reported by others (Adam, 1946; Keynes, 1952; Osserman,

* $\Delta \mathrm{A} / \mathrm{min}=$ delta absorbance/minute. It can be converted into SI units by a conversion factor calculated for the extinction coefficient of the reaction product $\left(1.36 \times 10^{4}\right)$ and the red cell count of the sample (see Ellman et al., 1961). 


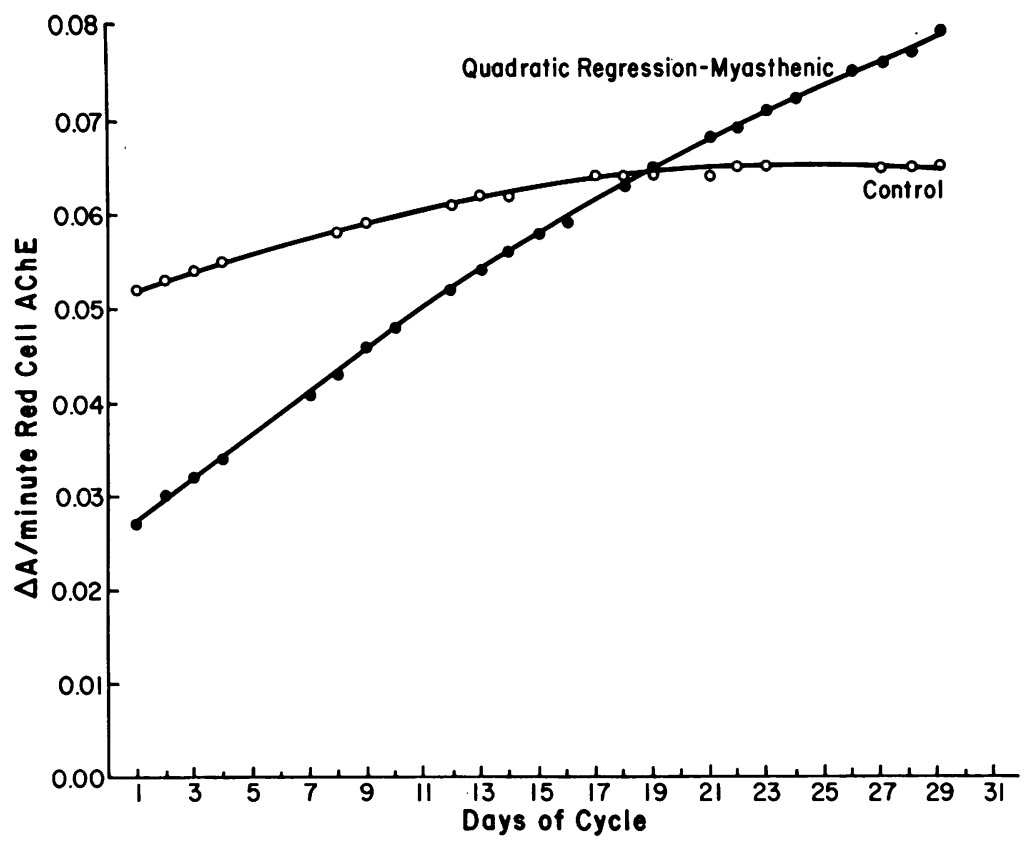

Fig. 2 Red cell AChE activity in myasthenic patients and control subjects compared by quadratic regression.

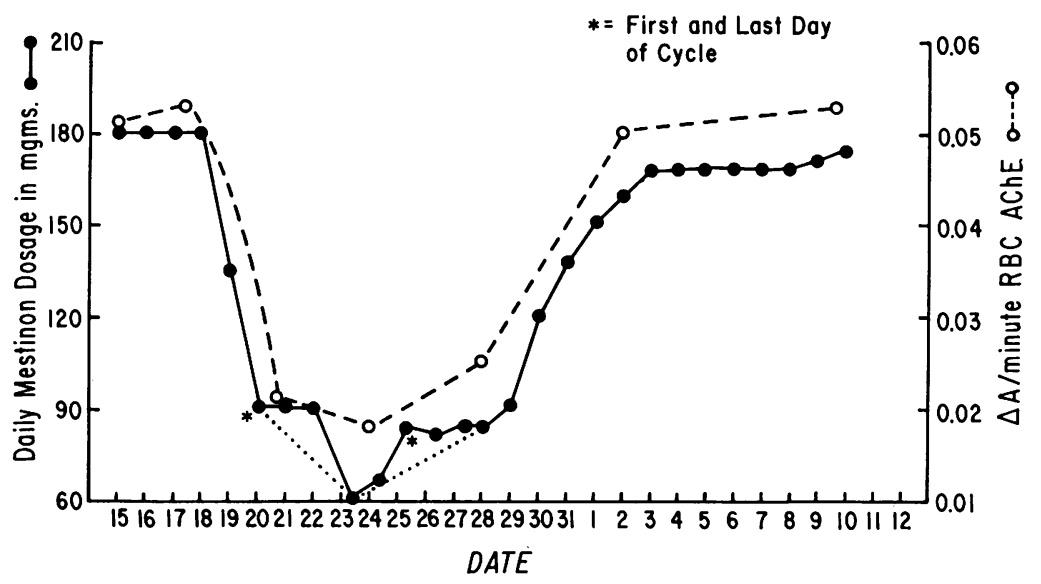

Fig. 3 Comparison of red cell AChE activity and medication requirement during a single menstrual cycle in the first myasthenic patient three months after thymectomy.

1958). A review of these cases indicates that 34$50 \%$ of these patients had deterioration of symptoms a few days before the onset of menstrual periods and subsequent improvement with the beginning of menstrual flow. This is very similar to the cyclical deterioration and improvement noted in three of 12 patients seen in our clinic.

It is reasonable to believe that the menstrual improvement of symptoms is related in some way to hormonal changes occurring during menstrua- tion. However, to date, the mechanism underlying this type of hormonally related remission has remained elusive. The mechanism of action of gonadal secretion on target organs, including skeletal muscles, has been the subject of numerous studies (Mueller et al., 1972; Massa et al., 1975). Most investigators have concentrated on the effects of sex hormones on electrolyte distribution, or protein and carbohydrate metabolism. These influences bring about long-term changes in skeletal 
muscle structure and function, and, therefore, cannot be held responsible for the cyclical and rapidly occurring functional alterations seen in the myasthenic patients.

Another probably relevant observation is the 'blocking action' of progesterone on uterine contractions (Csapo, 1956, 1973). Maintenance of pregnancy is dependent upon the endogenous and placental progesterone which inhibits the contraction of the myometrium. The actual mechanism which maintains this blocking action is still unknown. If such a blocking action also involves skeletal muscles, the menstrual remissions seen here could be attributed to progesterone withdrawal.

A possible effect of oestrogens on cholinergic metabolism may also explain this relationship. There have been only a few studies dealing with this aspect of the problem, examining the influence of hormones on muscle contraction or on acetylcholine metabolism (Reynolds and Foster, 1939; Torda, 1943; Barnes and Epperson, 1952). Hormonal influence on acetylcholine metabolism has been determined by assaying the activity of cholinesterases in the blood. The results of these earlier studies have been inconclusive. Moreover, they are difficult to interpret since all forms of esterases, not AChE specifically, were estimated simultaneously without the use of specific inhibitors. Inhibitors were not available at that time or were not used. However, more recent animal observations have shown that sex hormones do influence the activity of various esterases (Wooley, 1963; Augustinsson and Henricson, 1970). It has also been shown that in the immature and adult animals, AChE activity in the central nervous system can be increased by the administration of oestrogens (Moudgil and Kanungo, 1973).

Results of the present study demonstrate that there is an exaggerated cyclical variation in red cell AChE activity in some myasthenic patients which correlates well with the remission of symptoms during a menstrual cycle. The progressive increase in the activity of the red cell AChE observed in this study seems to go hand in hand with the increasing secretion of oestrogens as the menstrual cycle progresses. Then as the menstrual flow begins there is a sudden drop in the enzyme activity. Progesterone levels also follow a pattern similar to that of oestrogen during the latter half of the cycle and may have a similar influence on AChE activity. However, much less is known about the effects of progesterone on AChE. Some studies have indicated an abnormal progesterone metabolism in myasthenics (Schrire, 1959).
The diminished requirement for anticholinesterase medication can very well be due to the decrease in the red cell AChE activity. It is difficult to ascertain at this point whether the changes in red cell AChE activity are associated with similar fluctuations in enzyme levels at the neuromuscular junction. Regardless of whether changes occur at the neuromuscular junction or not, reduced activity of red cell AChE will by itself make more of the drug available for its action at the neuromuscular junction, thus improving transmission. A good correlation has been previously reported between the therapeutic efficiency of anticholinesterases and red cell AChE enzyme activity (Foldes and Smith, 1966).

An alternative possibility is that of a cyclical variation of immune functions as a result of changes in circulating levels of steroids. This is of special interest because of the recent demonstration that myasthenia gravis is definitely an autoimmune disease resulting from the development of antibodies to acetylcholine receptor molecules (Lindstrom et al., 1976a). Antibody titres against the receptors were found to be consistently raised in $87 \%$ of patients with myasthenia gravis (Lindstrom et al., 1976b). Measurement of antibody titres during the menstrual cycles in these patients will be of help in answering this question.

The AChE-hormonal interrelationship observed here seems to be prominent only in those myasthenic patients who exhibit cyclical menstrual remissions. It seems to be dependent also on thymic activity. Keynes (1952) reported that his myasthenic patients ceased to have the menstrual variations after thymectomy. A similar change was observed in both of our patients who underwent thymectomy. The thymus is known to have its own endocrine regulatory functions, and it has been well established that this organ controls the activity of a number of endocrine glands, including the gonads (Luckey, 1973). The thymus has recently been reported to play a role in female sexual maturation (Basedovsky and Sorkin, 1974). The exact mechanism of this complex AChEgonadal-thymic interrelationship is not, however, very clear. The thymus could also be instrumental in bringing about these changes through its influence on the immune system.

Present observations indicate that at least one of the mechanisms for menstrual improvement of symptoms in myasthenia gravis is due to cyclical changes in the activity of AChE enzyme. These cyclical enzyme changes are probably induced by the hormonal changes occurring during the menstrual cycle, and appear to be thymicdependent. Further experimental studies to 
explore thymic-gonadal interrelationships and their influence on the enzymes involved in neuromuscular transmission seem warranted.

We would like to thank Dr Alexandre Todorov for his help in the statistical analysis of the data.

\section{References}

Adam, G. S. (1946). Myasthenia gravis and pregnancy. Journal of Obstetrics and Gynaecology, 55, 557-561.

Augustinsson, K. B., and Henricson, B. (1970). Sex hormone as a factor controlling the blood plasma arylesterase activity in rats. Experientia, 26, 793794.

Barnes, A. C., and Epperson, J. W. (1952). Studies in the cholinesterase hormone relationship. American Journal of Obstetrics and Gynecology, 63, 326331.

Basedovsky, H. O., and Sorkin, E. (1974). Thymus involvement in female sexual maturation. Nature, 249, 356-358.

Coates, P. M., and Simpson, N. E. (1972). Genetic variations in human erythrocyte acetylcholinesterase. Science, 175, 1466-1467.

Collins, J. (1897). Asthenic bulbar paralysis. International Medical Magazine, 5, 203-212.

Csapo, A. I. (1956). Progesterone block. American Journal of Anatomy, 98, 273-291.

Csapo, A. I. (1973). The uterus-Model experiments and clinical trials. In The Structure and Function of Muscle. Vol. II. Edited by G. H. Bourne. Academic Press: New York.

Ellman, G. L., Courtney, K. D., Andres, V., and Featherstone, R. M. (1961). A new and rapid colorimetric determination of acetylcholinesterase activity. Biochemical Pharmacology, 7, 88-95.

Foldes, F. F., and Smith, J. C. (1966). The interaction of human cholinesterases with anticholinesterases used in the therapy of myasthenia gravis. Annals of the New York Academy of Sciences, 135, 287301.

Keynes, G. (1952). Obstetrics and gynaecology in relation to thyrotoxicosis and myasthenia gravis. Journal of Obstetrics and Gynaecology, 59, 173182.
Lindstrom, J. M., Lennon, V. A., Seybold, M. E., and Whittingham, S. (1976a). Experimental autoimmune myasthenia gravis and myasthenia gravis: Biochemical and immunochemical aspects. Annals of the New York Academy of Sciences, 274, 254274.

Lindstrom, J. M., Seybold, M. E., Lennon, V. A., Whittingham, S., and Duane, D. D. (1976b). Antibody to acetylcholine receptor in myasthenia gravis. Neurology (Minneapolis), 26, 1054-1059.

Luckey, T. D. (1973). Thymic Hormones. University Park Press: Baltimore.

Massa, E. M., Morero, R. O., Bloj, B., and Farias, R. N. (1975). Hormone action and membrane fluidity: Effect of insulin and cortisol on the Hill coefficients of rat erythrocyte membrane bound acetylcholinesterase and $\left(\mathrm{Na}^{+}\right.$and $\left.\mathrm{K}^{+}\right)$-ATP-ase. Biochemical and Biophysical Research Communications, 66, 115-122.

Moudgil, N. K., and Kanungo, M. S. (1973). Effect of age of the rat on induction of acetylcholinesterase of the brain by 17- $\beta$-estradiol. Biochemica Biophysica Acta, 329, 211-220.

Mueller, G. C., Vanderhaar, B., Kim, U. H., and Mahieu, H. L. (1972). Estrogen action: An inroad to cell biology. Recent Progress in Hormone Research, 28, 1-50.

Osserman, K. E. (1958). Myasthenia Gravis, p. 90. Grune and Stratton: New York.

Reynolds, S. R. M., and Foster, F. T. (1939). Relative cholinergic effects of selected estrogens. American Journal of Physiology, 128, 147-153.

Schrire, I. (1959). Progesterone metabolism in myasthenia gravis. Quarterly Journal of Medicine, 28, 59-75.

Torda, C. (1943). Effect of hormones on contraction of striated muscle and on cholinesterase activity. Proceedings of the Society for Experimental and Biological Medicine, 53, 121-125.

Viets, H. R., Schwab, R. S., and Brazier, M. A. B. (1942). The effect of pregnancy on the course of myasthenia gravis. Archives of Neurology and Psychiatry (Chicago), 47, 1082-1084.

Wooley, D. E. (1963). Sex differences in brain pseudocholinesterase activity in the rat. Journal of Neurochemistry, 10, 447-452. 